This item was submitted to Loughborough's Research Repository by the author.

Items in Figshare are protected by copyright, with all rights reserved, unless otherwise indicated.

\title{
Laser vibrometry meets laser speckle
}

PLEASE CITE THE PUBLISHED VERSION

PUBLISHER

(C) 2004 Society of Photo-Optical Instrumentation Engineers.

VERSION

VoR (Version of Record)

LICENCE

CC BY-NC-ND 4.0

REPOSITORY RECORD

Rothberg, Steve, and Ben J. Halkon. 2019. "Laser Vibrometry Meets Laser Speckle”. figshare. https://hdl.handle.net/2134/5837. 


\title{
Laser vibrometry meets laser speckle
}

\author{
Steve Rothberg* and Ben Halkon \\ Wolfson School of Mechanical and Manufacturing Engineering, Loughborough University,
}

\begin{abstract}
This paper begins with a review of the fundamental mechanism by which speckle noise is generated in Laser Vibrometry before describing a new numerical simulation of speckle behaviour for prediction of noise level in a real measurement. The simulation data provides real insight into the phase and amplitude modulation of the Doppler signal as a result of speckle changes. The paper also includes experimental data looking at the influence of speckle noise in measurements on rotors with a selection of surface treatments and in scanning and tracking configurations.
\end{abstract}

Keywords: laser vibrometry, laser speckle, vibration measurement

\section{INTRODUCTION}

When a coherent laser beam is incident on a surface that is optically rough, i.e. the surface roughness is large on the scale of the laser wavelength, the component wavelets of the scattered light become dephased. This condition is satisfied by almost all of the surfaces likely to be encountered in engineering structures. The dephased, but still coherent, wavelets interfere constructively and destructively, thus resulting in a chaotic distribution in backscatter of high and low intensities, referred to as a "speckle pattern".

The phenomenon of laser speckle was first reported by researchers working with the very earliest continuous wave lasers in the early 1960s. They talked about a "remarkable granular or peppery nature not present in ordinary light"1, "a sparkling appearance", and, less dramatically, "random dark and light spots"3. Much early work on laser speckle, described as "Enemy Number One" by Gabor", was directed at eliminating its detrimental effect in holography until techniques such as Laser Speckle Photography ${ }^{5,6}$ began to show how the speckle effect could be used to advantage in metrology. Since the early 1970s, applications in so-called 'Speckle Metrology"7 have been numerous.

Vibration measurements using Laser Vibrometers have now started to become commonplace. Users tend to be vibration engineers rather than optical specialists and applications have tended to be challenging. As a consequence of both these factors, speckle effects have begun to establish themselves as the crucial influence on Vibrometer performance.

Laser Vibrometers reported in the scientific literature have differed principally by the method used to produce the reference beam frequency shift and also in the interferometric arrangement used. It was in a design incorporating a rotating scattering $\operatorname{disc}^{8}$ that the effects of the speckle phenomenon first became apparent in Laser Vibrometry. While this design of Vibrometer has now been superseded, the range of likely applications has now increased to include a variety of measurements directly from rotating structures such as magnetic discs ${ }^{9,10}$, bladed discs ${ }^{11,12}$ and modal analysis on rotating discs ${ }^{13}$ and it is in such measurements where the effects of laser speckle are most apparent and most problematic. These applications use single beam Vibrometers which are suitable for translational vibration measurement. Rotational vibration measurements - torsional, pitch, yaw and roll vibrations - require parallel beam arrangements ${ }^{14}$ and these 'Rotational Laser Vibrometers' are also now available commercially. Measurements are not limited to rotating structures but they are routinely used on rotors for applications including assessment of torsional damper health ${ }^{15}$ or crankshaft bending vibration ${ }^{16}$. Since measurements with these devices are so likely to be made on rotating structures, users have found the influence of laser speckle to be particularly undesirable.

Figure 1 shows a schematic diagram of the interferometer that will be used to gather experimental data in a later part of this study. The rotating diffraction grating is used to divide the laser beam and to introduce a frequency shift between the

* s.j.rothberg@lboro.ac.uk; http://myprofile.cos.com/S234021ROh; http://www.lboro.ac.uk/departments/mm; phone +44(0)1509 227524; fax +44(0)1509 227615; Wolfson School of Mechanical and Manufacturing Engineering, Loughborough University, Loughborough, LEICS., LE11 3TU, U.K. 
two resulting beams. The lens makes the beams parallel for convenience and a large polarising beam splitter directs the beams to either target or reference surfaces. The quarter-wave plate ensures that light initially reflected at the beamsplitter is transmitted on scattering from the surfaces, after which the mirror / beamsplitter arrangement recombines them on the photodetector. Components are positioned such that optical paths are exactly matched. Where consideration of the effect of collecting multiple speckles on the photodetector is made, this is achieved experimentally simply by increasing the distance between the second beamsplitter and the photodetector. This is the configuration modelled in the numerical simulation reported here.

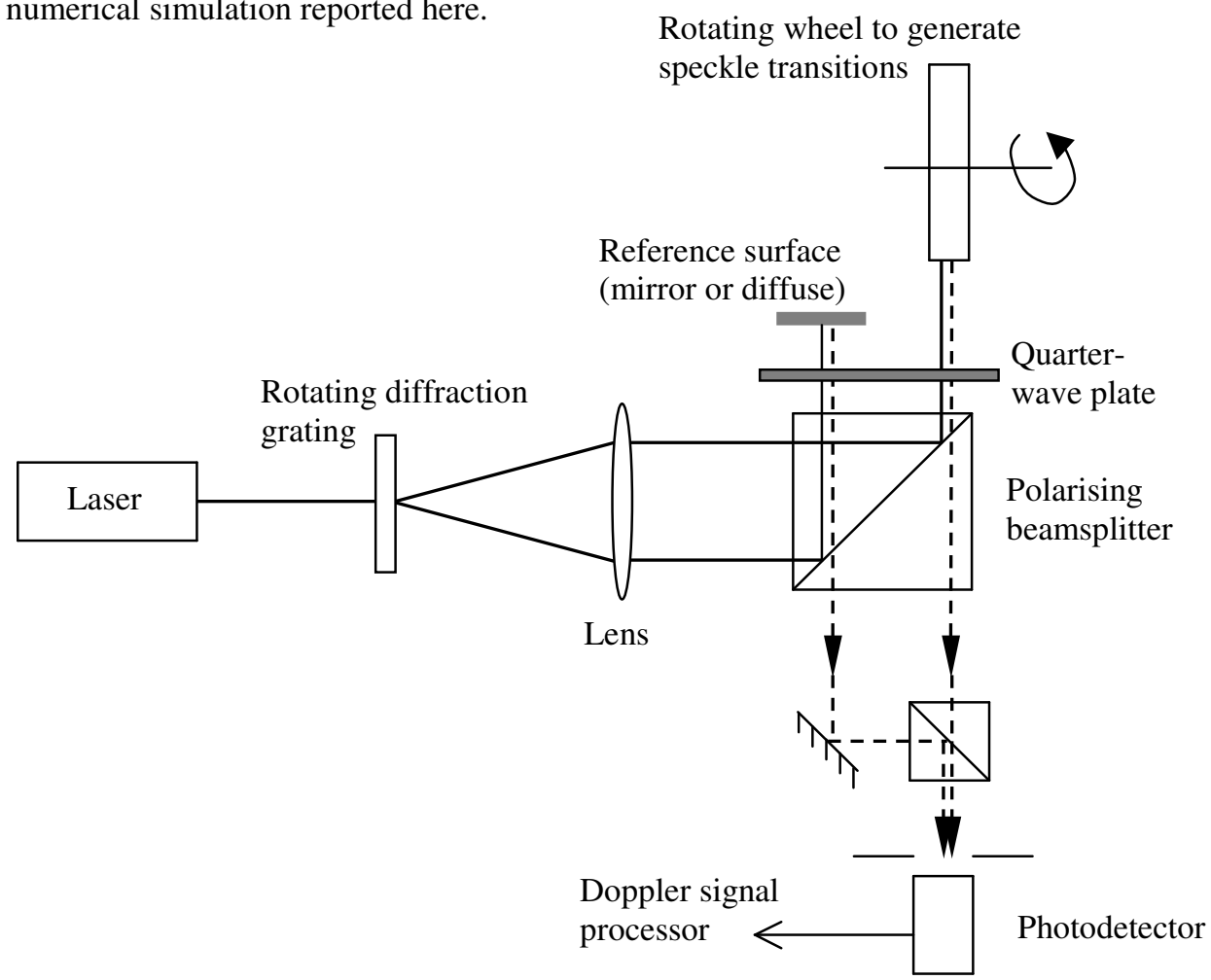

Figure 1: Schematic diagram of the experimental interferometer

\section{LASER SPECKLE AND PSEUDO-VIBRATION}

Figures $2 \mathrm{a} \& \mathrm{~b}$ show, respectively, the forms of the speckle patterns generated by scattering from a diffuse surface and by scattering from the retro-reflective tape that is often used with Laser Vibrometers as surface treatment. The speckle pattern produced by scattering from retro-reflective tape has a very distinctive Airy disc form as a result of diffraction at the glass beads on the surface of the tape. Statistically the speckle phases are uniformly distributed between $-\pi$ and $\pi$ and, neglecting the intensity distribution of the Airy disc, speckle intensities have a negative exponential probability distribution $^{17}$.

In the interferometer, the detected intensity, $I$, is given by the time-average of the square of the total light amplitude resulting from combination of target and reference beams. In its standard form this can be written ${ }^{18}$ :

$I=\bar{I}+\Delta I=\left(I_{R}+I_{T}\right)+2 \sqrt{I_{R} I_{T}} \cos \left[\left(2 \pi f_{R} t-2 k a(t)\right)+\left(\phi_{R}-\phi_{T}\right)\right]$

where $I_{R}$ and $I_{T}$ are the reference beam and target beam intensities respectively, $\phi_{R}$ is the reference beam phase across the detector and $f_{R}$ is its frequency shift, $\phi_{T}$ is the target beam phase at the detector if the target surface were stationary, $k$ is the light wavenumber and $a(t)$ is the target vibration displacement. The intensity sum, $\bar{I}=\left(I_{R}+I_{T}\right)$, is of no value in the vibration measurement and is usually filtered in some way. The second and relatively higher frequency term is generally referred to as the "Doppler signal" and is the component which, on demodulation, yields the time derivative of the target vibration displacement, i.e. the vibration velocity. 


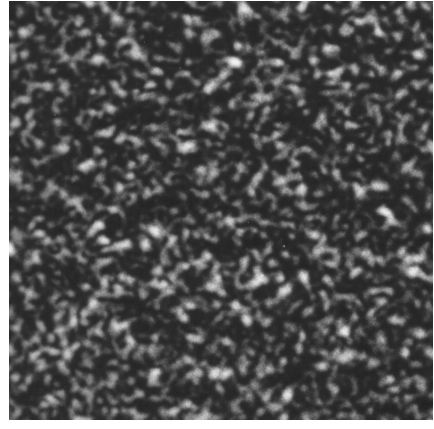

a) By scattering from a diffuse surface

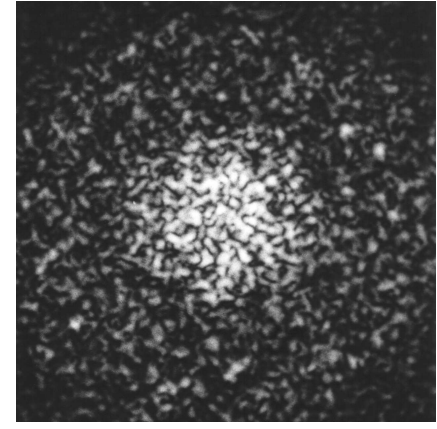

b) By scattering from retro-reflective tape

Figure 2: Speckle patterns

In reality, at least one of the beams incident on the photodetector takes the form shown in figure 2 with the photodetector sampling a small region within the speckle pattern. The Doppler signal, as described in equation (1), is therefore the result of a summation of speckles. This is usually of little consequence unless the speckles start to move or evolve in response to target motions. When this happens, there are two important effects on the Doppler signal. The first is an amplitude modulation and the second is a phase modulation, both of which result from the summation on the photodetector being performed over a changing population of speckles. Following equation (1), the Doppler signal component can be written:

$$
\Delta I=I_{\text {res }}(t) \cos \left[\left(2 \pi f_{R} t-2 k a(t)\right)+\Phi_{\text {res }}(t)\right]
$$

where $I_{\text {res }}$ and $\Phi_{\text {res }}$ are the time-varying resultant amplitude and phase of the Doppler signal. The amplitude modulation gives Doppler signals their characteristic appearance. It is possible for the Doppler signal amplitude to drop to a very low level in which case signal drop-outs can occur and glitches appear in the Laser Vibrometer output, contributing significant broadband noise. Often the importance of amplitude modulation is overemphasised, however, when considering the effects of laser speckle. Generally, the signal amplitude will remain at an acceptable level and phase modulation will be the major concern. The effect of the Doppler signal phase modulation in the Laser Vibrometer output is very clear. Figure 3 shows the form of the Laser Vibrometer output from a "measurement" on a non-vibrating, rotating target. This is "speckle noise". Figure 4 shows typical experimental data from the arrangement in figure 1, comparing speckle noise (low-pass filtered at $20 \mathrm{kHz}$ ) and the (normalised) Doppler signal RMS. Note, in particular, how speckle noise increases at low target-detector separations where Doppler signal RMS is especially high, emphasising how speckle noise is not a simple function of Doppler signal amplitude.

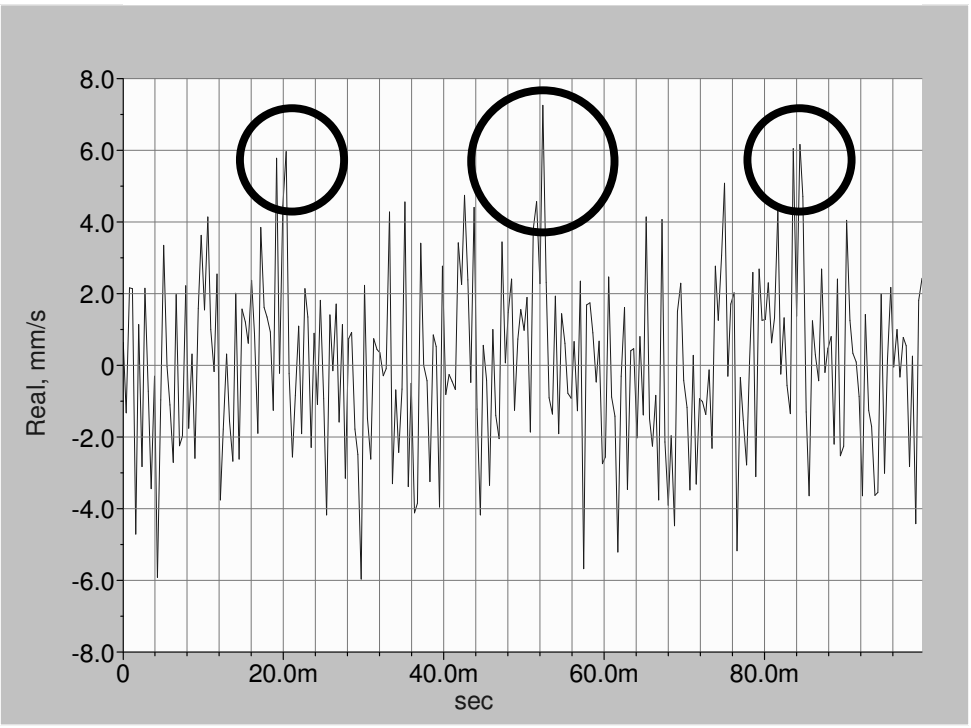

Figure 3: Laser Vibrometer output showing significant speckle noise

The frequency of the Doppler signal is known as the "beat frequency", $f_{\text {beat }}$, and is given by the modulus of the time derivative of the cosine argument in equation (2):

$$
f_{\text {beat }}=\left|f_{R}-\frac{2}{\lambda} \frac{d a(t)}{d t}+\frac{1}{2 \pi} \frac{d \Phi_{\text {res }}(t)}{d t}\right|
$$


where $\lambda$ is the laser wavelength. The frequency content of $\left(d \Phi_{\text {res }} / d t\right)$ will obviously appear in the output spectrum and this is worthy of further consideration.

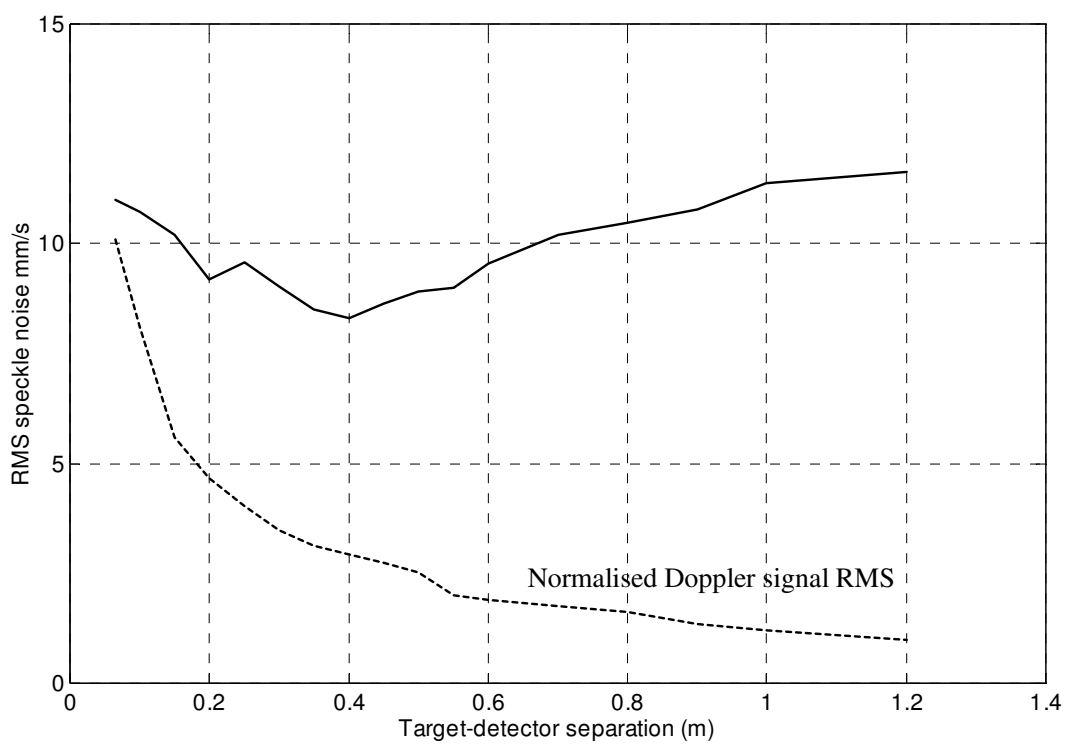

Figure 4: Variation of Doppler signal RMS and speckle noise with target-detector separation

If the speckle pattern changes are induced by nonnormal target motions, such as tilt, in-plane motion or rotation, which, in general, will be periodic with the same fundamental frequency as the on-axis vibration, then $\left(d \Phi_{\text {res }} / d t\right)$ will be pseudo-random in nature with the same fundamental frequency as the on-axis vibration. The periodic nature of the speckle noise is apparent in figure 3 , which relates to a "measurement" on a rotating, non-vibrating target, in which the added circles are intended to aid the reader in observing the periodicity. The characteristic spectrum of a pseudo-random signal consists of approximately equal amplitude peaks at the fundamental frequency and higher order harmonics. This additional signal content, first described as pseudo-vibration $^{18}$, will be indistinguishable from the genuine vibration information ${ }^{19}$. The spectrum of the data in figure 3 is shown in figure 5.

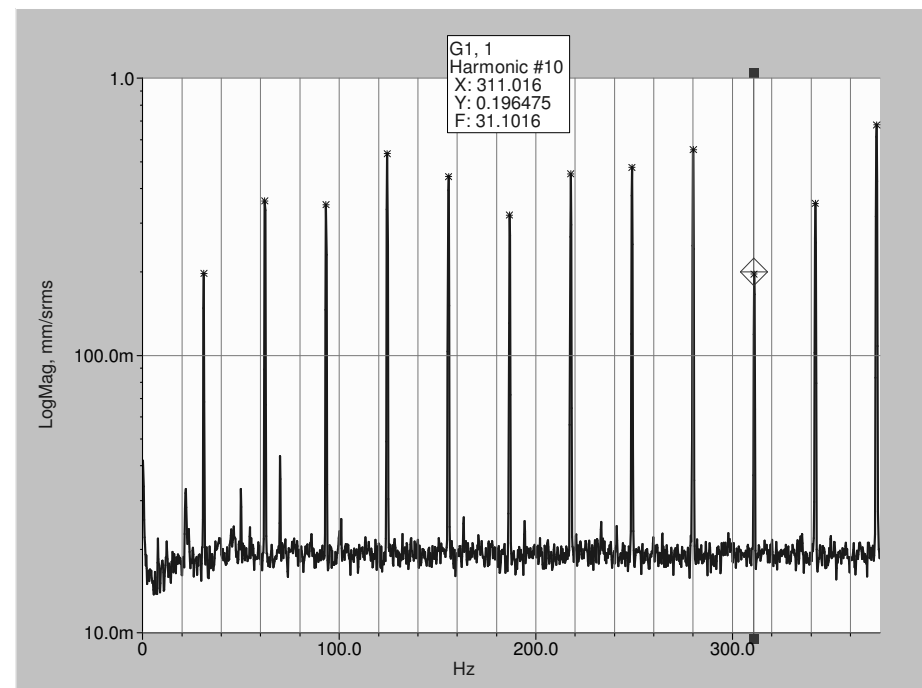

Figure 5: Laser Vibrometer output spectrum showing pseudo-vibration

The strength of the harmonic peaks serves to emphasise the periodicity of the output signal and a typical characteristic of the speckle noise generated in measurements on rotating targets is the way that the amplitudes of the harmonic peaks are maintained up to very high frequencies. Figure 5 shows peaks up to $12 x$ fundamental frequency but the speckle peaks in this data maintained their amplitude well above 100x fundamental frequency. For targets generating less rapid speckle transitions, such as those with only in-plane motion, the speckle peaks are more likely to decrease within the first few harmonics, giving a spectrum with a similar appearance to that from a harmonic vibration with a small amount of harmonic distortion. In each case, the speckle noise is concentrated at the frequencies in which the vibration engineer is likely to be most interested and a certain degree of judgement is required when interpreting low level data obtained with a Laser Vibrometer. Whenever speckle transitions do occur, the speckle noise generated will dominate any other noise source in the instrument output and the low noise-floors often quoted by manufacturers will not be attainable. 


\section{NUMERICAL SIMULATION}

The intensity and phase of the Doppler signal resulting when $P$ target beam speckles, with phase $\phi_{T p}$, intensity $I_{T p}$ and area $A_{p}$ on the photodetector (total area $A$ ) are mixed with a uniform reference beam has been shown previously ${ }^{18}$. For more general cases, for example where the reference beam contribution is itself a speckle pattern, it is convenient to work with the quadrature components of each contribution to the Doppler signal and this is how the summations are performed within the numerical simulation. With $N$ reference beam speckles, each with phase $\phi_{R n}$, intensity $I_{R n}$ and area $A_{n p}$ overlapping the $P$ th target beam speckle and on the photodetector, the summations take the following forms:

$$
\begin{gathered}
I_{\text {res }}=\frac{1}{A} \sqrt{\left[2 \sum_{n=1}^{N} \sum_{p=1}^{P} A_{n p} \sqrt{I_{R n} I_{T p}} \sin \left(\phi_{R n}-\phi_{T p}\right)\right]^{2}+\left[2 \sum_{n=1}^{N} \sum_{p=1}^{P} A_{n p} \sqrt{I_{R n} I_{T p}} \cos \left(\phi_{R n}-\phi_{T p}\right)\right]^{2}} \\
\tan \Phi_{\text {res }}=\left[\left(2 \sum_{n=1}^{N} \sum_{p=1}^{P} A_{n p} \sqrt{I_{R n} I_{T p}} \sin \left(\phi_{R n}-\phi_{T p}\right)\right) /\left(2 \sum_{n=1}^{N} \sum_{p=1}^{P} A_{n p} \sqrt{I_{R n} I_{T p}} \cos \left(\phi_{R n}-\phi_{T p}\right)\right)\right]
\end{gathered}
$$

\subsection{Phase Changes During Speckle Transitions}

Early simulations examined the phase change resulting from whole speckle transitions across a photodetector. For the case of a single speckle on the photodetector that is entirely replaced by a second uncorrelated speckle, it is easy to show how the random distribution of speckle phases leads to a mean phase change over many transitions of zero and a variance of $\left(\pi^{2} / 3\right) \mathrm{rad}^{2}$. For more than one speckle, the mathematical complexity increases significantly and numerical simulation is necessary. The early simulations performed agreed with the theoretical prediction for the single speckle case and showed how the variance decreased with increasing ratio, $\mathrm{M}$, of detector size in the direction of speckle motion to speckle size, according to the empirical relationship ${ }^{20}$ :

$$
\operatorname{Var}\left[\Delta \Phi_{r e s}(t)\right]=\frac{\pi^{2}}{3 M^{0.85}}
$$

Combination of phase change data from the simulation with correlation times for dynamic laser speckles ${ }^{21}$ gave the first speckle noise predictions but these failed to give good agreement with experimental levels.

This work had concentrated on the phase change in the Doppler signal during a single speckle transition and implicitly assumed that the rate of change of phase was constant throughout the transition. This is not, in fact, the case and the maximum rate of change is significantly underestimated by using the phase change across the full transition. In the numerical model this, therefore, introduces the need to sub-divide each speckle transition and 40 sub-divisions have been used.

\subsection{Effects of Wavefront Curvature}

For the arrangement shown in figure 1, simple consideration of the phase change due to speckle transitions, as described in the previous section and indicated in equation (5), would lead to the conclusion that minimum noise will result when a maximum number of speckles are collected i.e. at the closest target-detector separation possible. This is not borne out in figure 4, however, where speckle noise increases significantly close to the target. This increase is the result of wavefront curvature. In terms of speckle behaviour, the speckles move towards and then away from the target as they translate across the detector surface. The distance travelled is particularly significant at small target-detector separations. For this reason, the simulation breaks each speckle into a $W \mathrm{x} H$ grid, typically 40x10, in order to apply a curvature correction to the speckle phase.

\subsection{Structure of the Numerical Simulation}

The simulation has been written to model the speckle noise resulting when the target laser beam is incident in a radial direction on the circumference of a rotating (nominally circular cross-section) wheel as shown in figure 1 . The number of speckle transitions is also adjusted to ensure that the number of data points generated in the simulation is always a power of 2 for optimum FFT efficiency. A typical value in excess of 3200 (around 128000 partial speckle transitions) was used 
in the simulation. In terms of the experimental arrangement used, this is approximately equivalent to the speckle transitions occurring during half a rotation cycle.

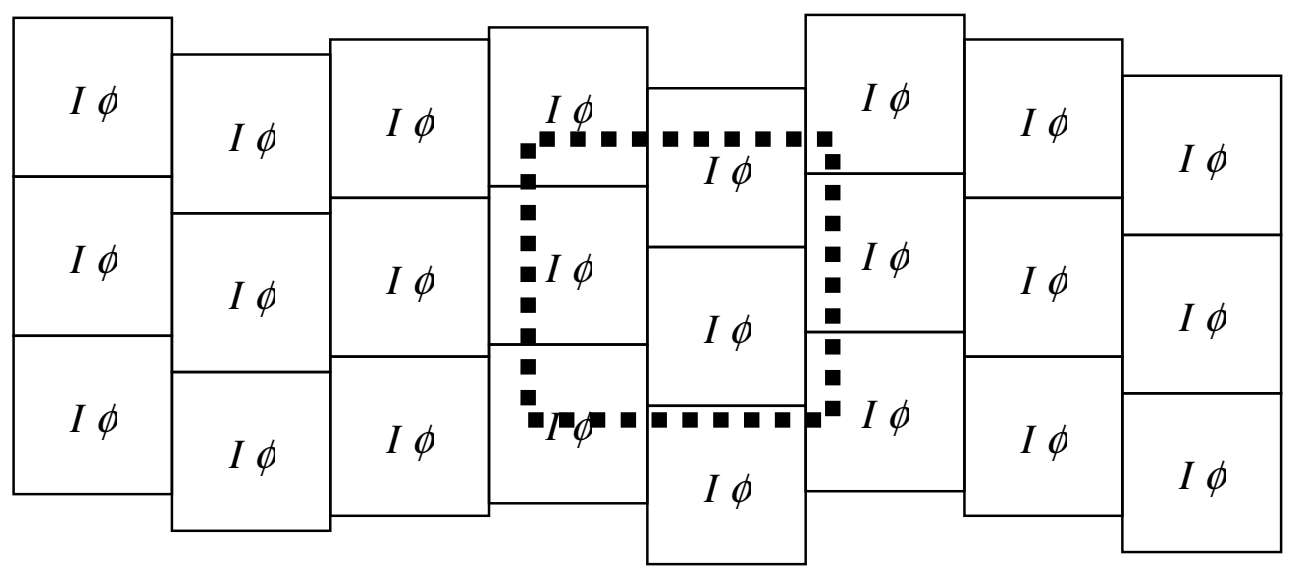

Speckle Motion

Figure 6: Representation of the 'target' speckle pattern matrix.

Figure 6 is intended to help visualise the physical significance of the 'target' speckle pattern matrix generated. The figure shows, in heavy dashed lines, the fixed position of a photodetector whose dimensions ( $L_{w}$ by $L_{h}$ ) are, in this case, 2 speckles by 2 speckles. The speckles translate across the photodetector surface from left to right. In the existing simulation only speckle translation, rather than boiling ${ }^{21}$, is considered as this is reasonable for the configuration under scrutiny. Each speckle has intensity with a negative exponential probability density generated from a random number, $x_{p}$, as follows:

$\frac{I_{T_{p}}}{\langle I\rangle}=-\ln \left(\frac{1-x_{p}}{1+(1 \mathrm{e}-10)}\right)$

where $\langle I\rangle$ is the mean speckle intensity and the quantity $1 \mathrm{e}-10$ is added to the denominator of the $\ln$ argument to prevent an attempt to evaluate $\ln (0)$ while limiting distortion of the probability distribution. Speckles are assigned phases in the range $-\pi$ to $\pi$ using an independent sequence of random numbers to that used to generate intensity values. The 'width' of the matrix, i.e. in the direction of speckle motion, is determined by the number of full speckle transitions over which the simulation is to be performed. The 'height' of the matrix is set by the maximum number of speckles collected in this dimension i.e. at the shortest target-detector separation.

For the purposes of the later inclusion of wavefront curvature and to ensure accuracy in the rate of change of phase calculation, each speckle is then divided into a $W \mathrm{x} H$ grid. This also conveniently allows disruption of what would otherwise be a very regular presentation of each 'speckle' to the detector without attempting to replicate the full irregularity associated with speckle shapes. The variation in the position of the speckle on the detector, as shown in figure 6 , is limited to $H$ steps.

The reference beam contribution can be either of uniform intensity or as a stationary speckle pattern. If the latter, then the arrangement of speckles on the detector is also disrupted. In this case, the rows (rather than the columns) of the speckle matrix are misaligned ( $W$ randomly selected possible positions) and the columns are set so that partial speckles are collected at the top and bottom of the detector.

Once the intensity and phase matrices are complete for the target and reference beam contributions, the simulation moves into its outer loop which controls the number of speckles incident on the photodetector for each completion of the inner loop of the simulation. Experimentally (see figure 1), this is analogous to varying the target-detector separation and for the range used in the simulation (1 to 20 speckles equal to the photodetector width, taken in 10 steps), separations between $10 \mathrm{~cm}$ and $1.2 \mathrm{~m}$ are simulated. 


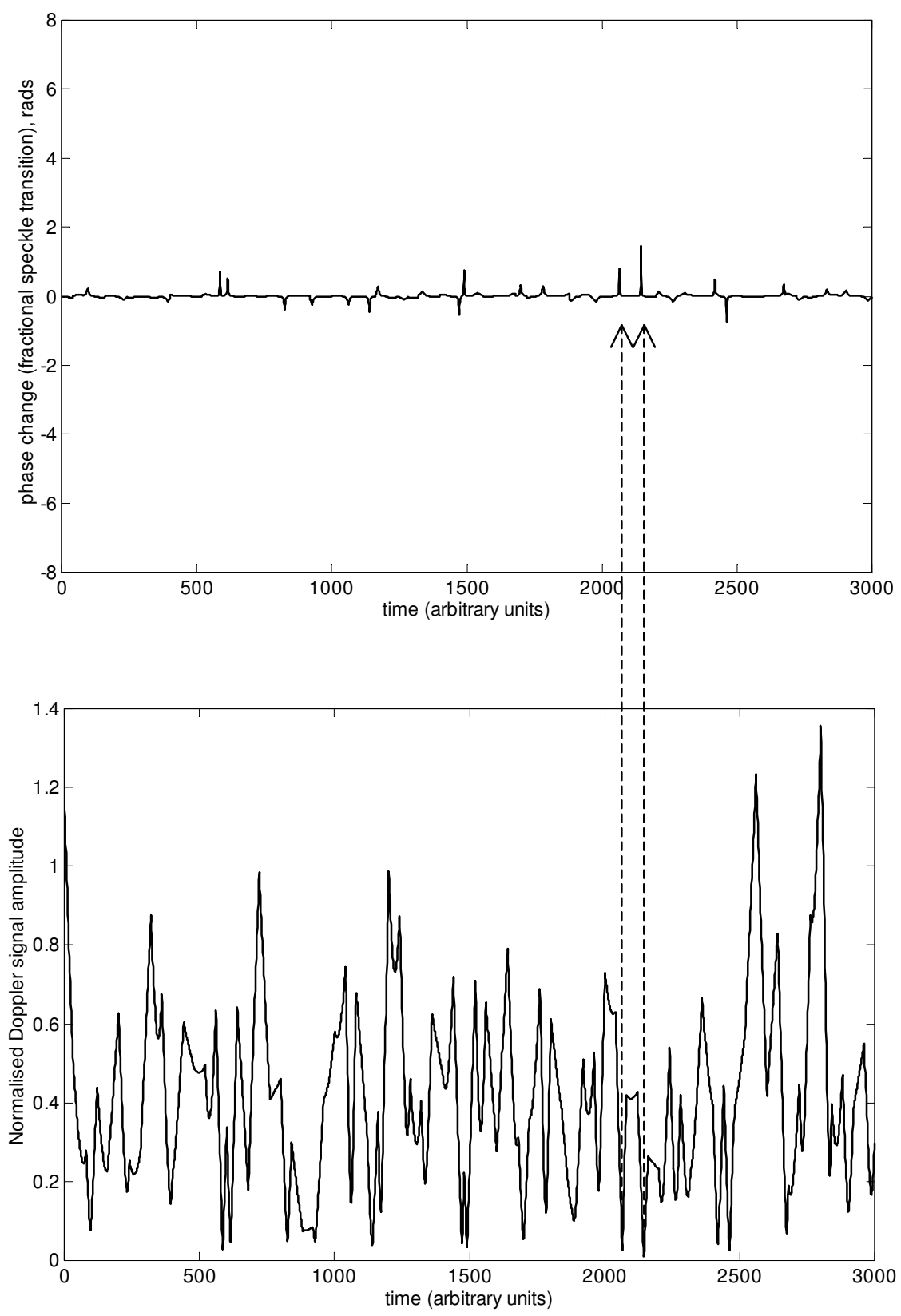

Figure 7: Simulated phase change data after removal of $2 \pi$ wraparounds and the effect of low Doppler signal amplitude

The inner loop calculates the Doppler signal quadrature components after every partial speckle transition. At this stage, the target and reference beam contributions on the detector are in the form of matrices with dimensions $(M W) \times(\alpha M H)$ where $\alpha$ is the aspect ratio of the photodetector. The products $2 \sqrt{I_{R} I_{T}} \cos \left(\phi_{R}-\phi_{T}\right)$ and $2 \sqrt{I_{R} I_{T}} \sin \left(\phi_{R}-\phi_{T}\right)$ are first calculated for the corresponding elements in these matrices, before summation of each component across the whole detector.

The quadrature components are used to provide Doppler signal amplitude and phase according to equations (4a\&b) for each speckle summation and, from this, the change in phase follows straightforwardly. At this initial stage, the phase change data is dominated by $2 \pi$ wraparounds. These are artefacts of the simulation and are corrected by running the phase change matrix through a loop to detect phase changes in excess of $\pi$ which is the maximum change possible in a single transition. The corrected phase change data are shown in figure 7 in which quite significant transient phase 
changes are still evident. Such a feature in the phase change data will have a significant effect on the speckle noise level across a broad frequency band. Further investigation reveals how these are related to periods of low signal amplitude, as also shown in figure 7 for two arbitrarily selected peaks in the phase change data. In fact, the signal amplitude need only be low relative to its mean level rather than in an absolute sense for this to occur since increasing all the speckle intensities by some factor will have no effect on the phase calculation. Laser Vibrometers sometimes use track-and-hold type circuits to contend with periods of low signal amplitude but these are likely to be relatively ineffective in limiting this noise generating mechanism. The full simulation incorporates a track-and-hold simulation so that the effect on speckle noise can be observed.

The speckle noise prediction requires rate of change of phase so the time taken for the partial transition, $T_{w}$, is required $^{22}$. For the case of a laser beam incident with $\exp [-2]$ diameter $D$ and radius of curvature $r$ on a target of radius $R$ and rotation angular frequency $\Omega$ producing speckle with size $\left\langle\sigma_{0}\right\rangle$ on a photodetector at distance $z$ from the target:

$T_{w}=\frac{\left\langle\sigma_{0}\right\rangle}{W \Omega}\left[2\left(\left(\frac{\left\langle\sigma_{0}\right\rangle R}{D}\right)^{2}+\left(R\left(1+\frac{z}{r}\right)+2 z\right)^{2}\right)\right]^{-1 / 2}$

The time taken for speckle transitions is typically very short. In supporting experimentation in which target rotation was at $30 \mathrm{~Hz}$, equation (7) indicates that the time for a full speckle transition would be in the region of $2 \mu \mathrm{s}$. As a result, the speckle noise generated extends across an extremely broad frequency range, certainly well beyond the frequency range of general interest to a vibration engineer making a measurement of this kind. It is, therefore, essential to include a filter in the simulation set to the upper limit in the corresponding experimental analysis. The simulation now proceeds by calculating the FFT of the speckle noise so that the noise measured in any chosen frequency range can be calculated.

\subsection{Results of the Simulation}

Figure 8 shows typical results from the simulation for the case of a uniform intensity reference beam contribution and in the absence of correction for wavefront curvature. The trend demonstrated in equation (5) causes a reduction in speckle noise with decreasing target-detector separation in the predicted data, both in total level and after low pass filtering at $5 \mathrm{kHz}$ where a general trend of decreasing speckle noise with decreasing target-detector separation is observed. In experimental data, such as that shown in figure 4, the opposite trend is observed at close target-detector separations and wavefront curvature is responsible for this as demonstrated by figure 9 .

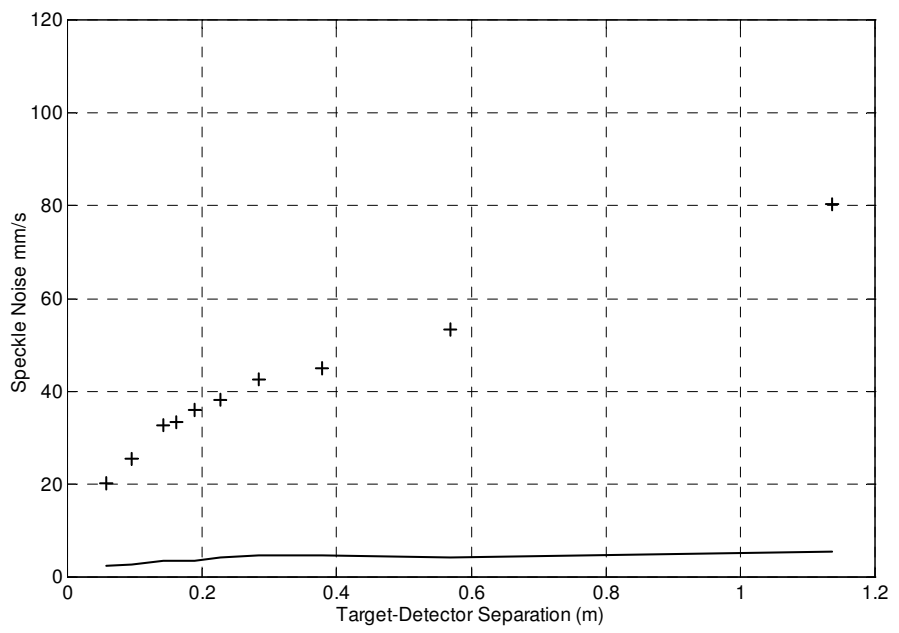

Figure 8: Speckle noise prediction (uniform intensity reference beam contribution, no curvature correction). Total noise (+), Noise low-pass filtered at $5 \mathrm{kHz}$ (solid line).

Figure 9 shows typical results from the simulation for the case of a uniform intensity reference beam contribution with adjustments made for wavefront curvature. The total speckle noise has an RMS value in the range $60-90 \mathrm{~mm} / \mathrm{s}$. Such a high noise level would be prohibitive for a real measurement but recall that this is across a much broader frequency 
range than that of interest in this type of application. In the filtered data (upper frequency limit again $5 \mathrm{kHz}$ ), a considerable reduction in RMS speckle noise occurs, down to $4-7 \mathrm{~mm} / \mathrm{s}$ which is tolerable for such applications. By comparison with figure 8 , the data shows how, at close target-detector separations (up to $0.4 \mathrm{~m}$ which represents a detector size in the direction of speckle motion equal to 3 speckles), the effect of wavefront curvature is to increase significantly the variance of the phase change associated with speckle transitions leading to high speckle noise levels. These levels reduce with increasing separation as both the transition time increases and the wavefront curvature effects become less prominent. Above $0.6 \mathrm{~m}$ (representing a detector size in the direction of speckle motion equal to 2 speckles), the trend follows that of the data in figure 8 under the influence of fewer speckles being collected on the detector. The track-and-hold facility has little effect except at the greater target-detector separations where only a few relatively low intensity target beam speckles are collected. This is because the uniform reference beam gives a strong Doppler signal whose amplitude rarely drops below the pre-set threshold. In the context of a real measurement, the prediction indicates that, after low-pass filtering at $5 \mathrm{kHz}$, there is not a large variation with target-detector separation in measured speckle noise but the data does show a minimum in the region $0.4-0.6 \mathrm{~m}$, representing a detector size in the direction of speckle motion equal to 2 to 3 speckles.

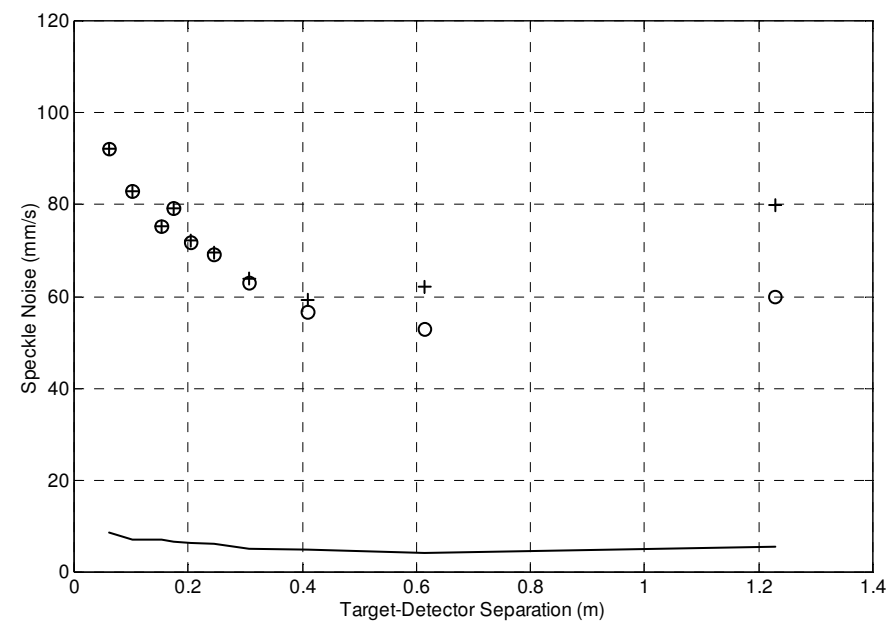

Figure 9: Speckle noise prediction (uniform intensity reference beam contribution).

Total noise (+), Total noise after track-and-hold (O), Noise low-pass filtered at $5 \mathrm{kHz}$ (solid line).

\section{EXPERIMENTAL DATA}

Experimental confirmation of the data shown in figure 9 is the subject of ongoing research but initial experimentation has confirmed the prediction of levels in the range $4-7 \mathrm{~mm} / \mathrm{s}(30 \mathrm{~Hz}$ rotation, $0-5 \mathrm{kHz})$. Attention is now turned to interesting speckle noise data gathered from the kinds of configuration that the simulation will go on to study.

\subsection{Effects of target surface}

In many cases, rotating targets have machined or polished surfaces, such that the "optically rough" diffuse backscattering condition, which is advantageous when making off-normal measurements (as rotor measurements generally are), is not met. In such cases it is necessary to treat the surface with some form of diffuse scatterer but this unavoidably results in speckle noise becoming significant. In figure 10, measurements from a number of typical surface treatments are shown: machined/polished surface (MS), retro-reflective tape (RT), white "sticky-backed" paper (WP), matt black spray paint (MBP) and developer spray (DS).

Figure 10 shows the noise for similar measurements using three commercial instruments on a rotating target with the various surface treatments. The average speckle noise power spectral densities are shown normalised to the largest value found in that group of values. For two of the Vibrometers, the noise levels experienced when making measurements on optically smooth surfaces are significantly different to those experienced with rough surfaces. It is believed that this significant difference is not associated with variations in speckle noise levels but is due to the mirror-like behaviour of 
the polished surface leading to loss of signal when low light levels are collected on the photodetector. Measurements on highly reflective rotating surfaces are therefore not recommended due to this unpredictability.

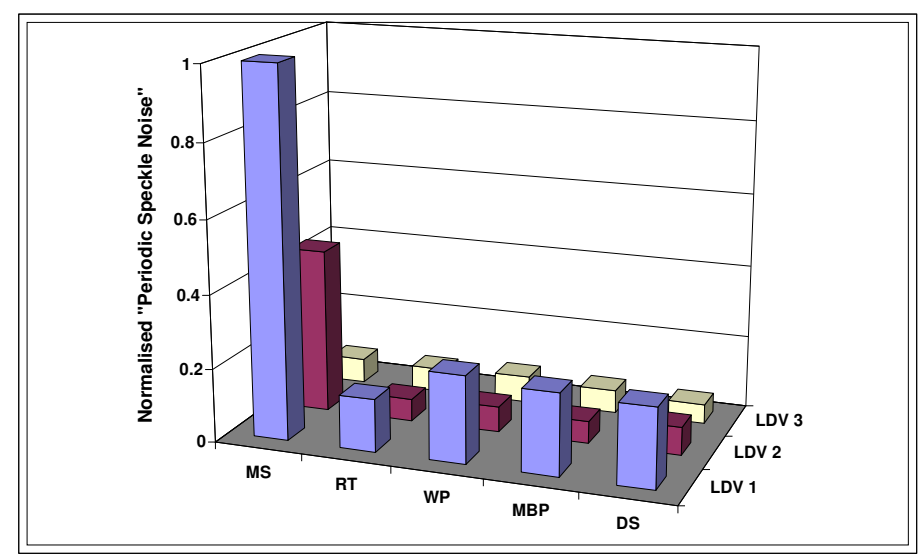

For all three instruments and all surface treatments, the differences in performance are within the uncertainty level of the calculation. The retroreflective surface is marginally the best surface to use but the difference compared to, for example, matt black paint, is not great. This result is of interest as it emphasises the fact that the speckle noise problem is not driven by signal amplitude problems.

Figure 10: speckle noise levels with different surface treatments

\subsection{Speckle noise in scanning and tracking measurements}

A stationary laser beam incident on a rotating target will result in speckle noise which repeats at the target rotation frequency whilst a scanning laser beam incident on a stationary target will result in a speckle noise repeat at the scan frequency. A scanning laser beam incident on a rotating target can give rise to speckle noise which repeats at a frequency other than the scan or the rotation frequencies ${ }^{23}$. In such a case, the speckle repeat has a period that corresponds to whenever both the scan and the rotation have completed integer numbers of cycles.

The velocity measured in such a measurement is illustrated in Figure 11 where a scan at $12.5 \mathrm{~Hz}$ combined with rotation at $10 \mathrm{~Hz}$ resulted in a speckle noise repeat at $2.5 \mathrm{~Hz}$, i.e. 5 cycles of scan and 4 cycles of rotation. The sharpness of these harmonic peaks and the high order up to which they prevail are classic characteristics of speckle noise. This is, of course, just one of the many speckle repeat possibilities and a full map of speckle repeat frequencies is shown in Figure 12. The lower limit apparent on the ratio of speckle repeat frequency / rotation frequency is equal to the resolution in the spectrum. In this data this has been set at 1/50th of the rotation frequency, i.e. the data length is equal to the time taken for 50 rotation cycles. The solid line shown corresponds to four times the resolution in the spectrum and, based on experience, is proposed as the lowest speckle repeat frequency that could be seen clearly in the spectrum.

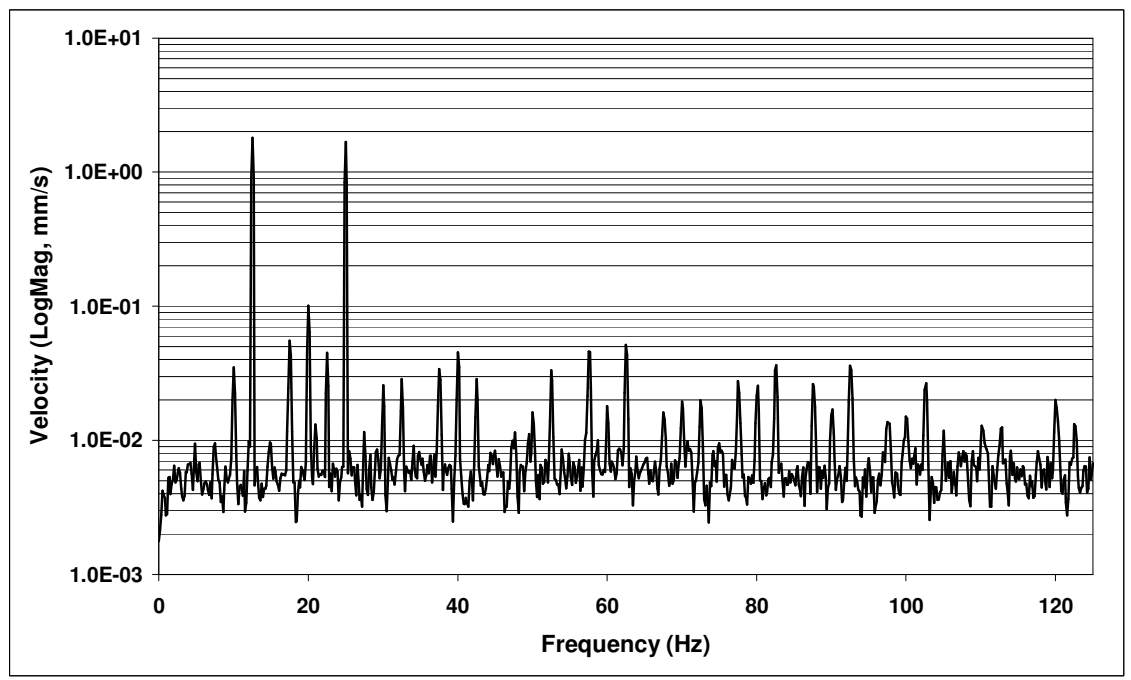

Figure 11: Velocity measured in a typical circular scanning Laser Vibrometer measurement on a rotating, non-vibrating target

The plot is dimensionless such that the LDV user could plot different limits on this particular map (for any resolution coarser than 1/50th of the rotation frequency). The data points above the solid line thus represent the repeat frequencies that can be seen at all the specific values of the ratio of scan frequency / rotation frequency at which repeats could be observed. These must obviously include the example shown previously in Figure 11 and this data-point is shown highlighted in a circle in Figure 12. 


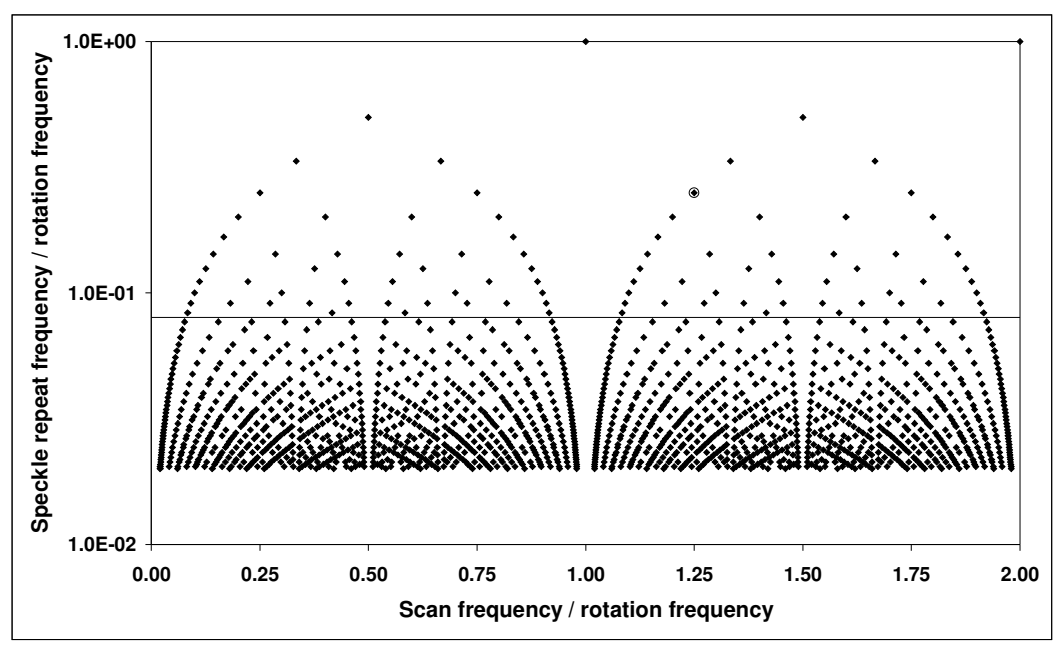

Figure 12: Speckle repeat map

The tracking condition, where scan frequency / rotation frequency $=1$, merits further discussion. The speckle repeat map shows that the speckle repeat frequency should equal rotation frequency at this condition and, if perfect tracking could be achieved, the speckle pattern on the detector might be expected to rotate but not to change its form, resulting in

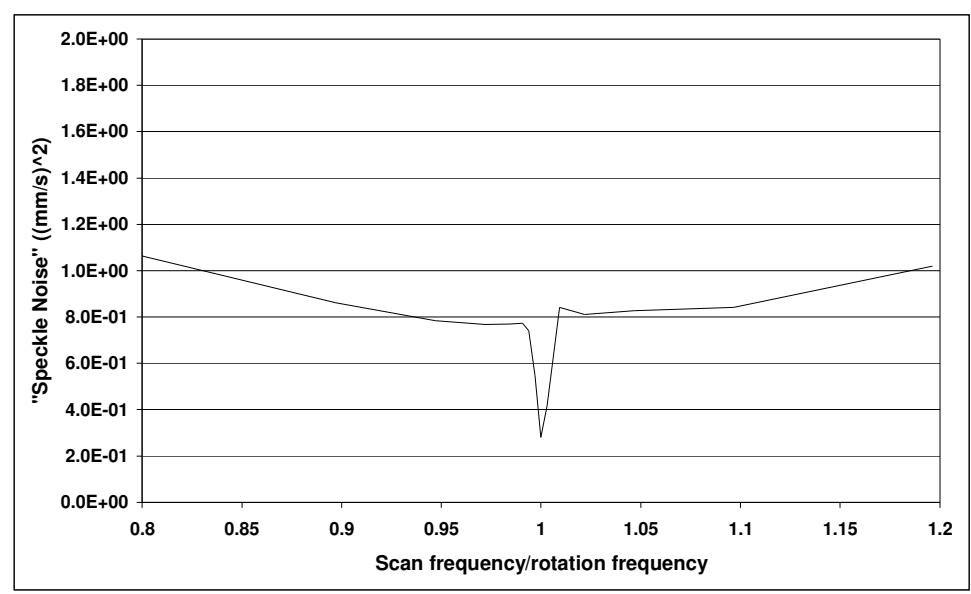
extremely low noise in the instrument output. In reality, as discussed earlier, there are small but inevitable misalignments between target rotation and optical axes, as well as imperfections in the scan profile, that mean there will still be modest changes in the collected speckle pattern ${ }^{23}$. Nonetheless, a significant drop in speckle noise does result as the tracking condition is approached and this is illustrated in Figure 13. Here, the spectral mean square noise when tracking is at least a factor of 2 down on that when the scan and rotation frequencies differ by just a few percent.

Figure 13: Speckle noise in circular scanning Laser Vibrometer measurements on rotating targets

\section{CONCLUSIONS}

This paper has given details of a how speckle noise manifests itself in Laser Vibrometers together with a numerical simulation to enable explanation of the underlying phenomena in speckle noise generation and ultimately prediction of measured levels. The particular application examined was that of a measurement on a rotating target since this represents a worst case scenario as far as speckle noise is concerned.

The simulations showed how the changing population of randomly phased speckles caused variation in the resultant Doppler signal phase as the fundamental mechanism. Speckle transitions across the photodetector were considered in increments of 1/40th of speckle size to prevent an underestimation of rate of change of phase that would otherwise occur. and corrections were applied to speckle phase for the effects of wavefront curvature which were shown to be extremely significant at short target-detector separations. The noise generated in speckle transitions was shown to extend across an extremely broad frequency range, much broader than is of interest to the engineer considering the application under scrutiny. A reduction of the order of a factor of 10 was found in the predicted RMS speckle noise after low-pass filtering at an appropriate frequency. A particularly important contribution to overall noise level was made by peaks in the phase change data resulting after periods of relatively low Doppler signal amplitude. These may not be eliminated by track- 
and-hold circuitry because signal amplitude may not have been 'low' in an absolute sense but 'low' relative to the mean signal level for a particular configuration. In initial experimental comparisons, good agreement was found between Doppler signal amplitude and speckle noise RMS.

Further experimental data has shown how speckle noise dominates the vibrometer output noise in measurements on rotors. Surface treatment is recommended with retro-reflective tape proving marginally the best option. Speckle noise in scanning measurements has also been discussed. The tendency to generate harmonic peaks at frequencies other than scan or rotation frequency has been explained and the reduction of speckle noise amplitudes when tracking has also been demonstrated experimentally.

\section{Acknowledgements}

The authors wish to acknowledge the support of the Engineering and Physical Sciences Research Council, the Leverhulme Trust and the Royal Society as well as the hospitality of the Dipartimento di Meccanica, Universita di Ancona where the first part of this work was completed.

\section{References}

1. J.D. RIGDEN and E.I. GORDON 1962 Proc I.R.E, 50, 2367-2368. The granularity of scattered optical maser light.

2. B.M. OLIVER 1963 Proc IEEE, 51, 220-221. Sparkling spots and random diffraction.

3. $\quad$ R.V. LANGMUIR 1963 Applied Physics Letters, 2(2), 29-30. Scattering of laser light.

4. D. GABOR 1970 IBM Journal of Research and Development, 509-514. Laser speckle and its elimination.

5. J.M. BURCH and J.M.J. TOKARSKI 1968 Optica Acta, 15(2), 101-111. Production of Multiple Beam Fringes from Photographic Scatterers.

6. M. FRANCON 1979 Laser speckle and applications in optics, Academic Press, New York.

7. $\quad$ R.K. ERF (Ed.) 1978 Speckle Metrology, Academic Press, New York.

8. N.A. HALLIWELL 1979 Journal of Sound and Vibration, 62, 312-315. Laser Doppler Measurement of Vibrating Surfaces: A Portable Instrument.

9. T.A. RIENER, A.C. GODING and F.E. TALKE 1988 IEEE Transactions on Magnetics 24(6), $2745-2747$. Measurement of head/disc spacing modulation using a two channel fiber optic laser Doppler vibrometer.

10. R.W. WLEZEIN, D.K. MIU and V. KIBENS 1984 Optical Engineering 24(4), 436-442. Characterization of rotating flexible disks using a laser Doppler vibrometer.

11. R.A. COOKSON and P. BANDYOPADHYAY 1980 Transactions of the ASME Journal of Engineering for Power 102, 607-612. A fiber-optics laser Doppler probe for vibration analysis of rotating machines.

12. A.K. REINHARDT, J.R. KADAMBI and R.D. QUINN 1995 Transactions of the ASME Journal of Engineering for Gas Turbines and Power 117, 484-488. Laser vibrometry measurements on rotating blade vibrations.

13. A.B. STANBRIDGE and D.J. EWINS 1995 Proceedings of ASME Design Engineering Technical Conference, Boston, USA 3(B), 1207-1213. Modal testing of rotating discs using a scanning LDV.

14. N.A. HALLIWELL, C.J.D. PICKERING and P.G. EASTWOOD 1984 J. Sound and Vibration 93(4), 588-592. The laser torsional vibrometer: a new instrument.

15. N.A. HALLIWELL and P.G. EASTWOOD 1992 Optics and Lasers in Engineering , 16(4/5), 337-350 'Marine Vehicles and Offshore Installations: Laser Diagnostics of Machinery Health.

16. J.R. BELL and S.J. ROTHBERG, 2000. Journal of Sound and Vibration, 238(4), 673-690. Rotational vibration measurements using laser vibrometry: comprehensive theory and practical application.

17. J.C. DAINTY, 1975. Laser Speckle and Related Phenomena, Springer-Verlag, Berlin.

18. S.J. ROTHBERG, J.R. BAKER and N.A. HALLIWELL, 1989. Journal of Sound and Vibration 135(3), 516-522. Laser vibrometry: pseudo-vibrations.

19. S.J. ROTHBERG and N.A. HALLIWELL 1994 Journal of Laser Applications 6(1), 38-41. Laser Vibrometry on Solid Surfaces: The Effects of Laser Speckle.

20. M. DENMAN, N.A. HALLIWELL and S.J. ROTHBERG, "Speckle Noise Reduction in Laser Vibrometry: Experimental and Numerical Optimisation". Proceedings of 2nd International Conference on Vibration Measurements by Laser Techniques, 1996, pp12-21, Ancona, Italy.

21. N. TAKAI, T. IWAI and T. ASAKURA 1981 Applied Physics B 26 185-192. An effect of curvature of rotating diffuse objects on the dynamics of speckle produced in the diffraction field.

22. I.D.C. TULLIS, N.A. HALLIWELL and S.J. ROTHBERG, 1998 Applied Optics, 37(30), 7062-7069. Spatially Integrated Speckle Intensity: Maximum Resistance to Decorrelation due to In-Plane Target Displacement.

23. B.J. HALKON, S.R. FRIZZEL and S.J. ROTHBERG, 2003 Meas. Sci. Technol., 14, 773-783. Vibration measurements using continuous scanning laser vibrometry: velocity sensitivity model experimental validation 